\title{
Crystal-MUSIC: Accurate Localization of Multiple Sources in Diffuse Noise Environments Using Crystal-Shaped Microphone Arrays
}

\author{
Nobutaka Ito $^{1,2}$, Emmanuel Vincent ${ }^{1}$, Nobutaka Ono $^{2}$, Rémi Gribonval ${ }^{1}$, and \\ Shigeki Sagayama ${ }^{2}$ \\ 1 INRIA, Centre de Rennes - Bretagne Atlantique \\ Campus de Beaulieu, 35042 Rennes Cedex, France \\ \{nobutaka.ito, emmanuel.vincent, remi.gribonval\}@inria.fr \\ 2 The University of Tokyo \\ 7-3-1 Hongo, Bunkyo-ku, Tokyo, Japan \\ \{ito, onono, sagayama\}@hil.t.u-tokyo.ac.jp
}

\begin{abstract}
This paper presents crystal-MUSIC, a method for DOA estimation of multiple sources in the presence of diffuse noise. MUSIC is well known as a method for the estimation of the DOAs of multiple sources but is not very robust to diffuse noise from many directions, because the covariance structure of such noise is not spherical. Our method makes it possible for MUSIC to accurately estimate the DOAs by removing the contribution of diffuse noise from the spatial covariance matrix. The denoising of the matrix (i.e. removal of the diffuse noise component) consists of two steps: 1) denoising of the off-diagonal entries via a blind noise decorrelation using crystal-shaped arrays, and 2) denoising of the diagonal entries through a low-rank matrix completion technique. The denoising process does not require the spatial covariance matrix of diffuse noise to be known, but relies only on an isotropy feature of diffuse noise. Experimental results with real-world noise show that the DOA estimation accuracy is substantially improved compared to the conventional MUSIC.
\end{abstract}

Key words: Diffuse noise, DOA estimation, microphone arrays, MUSIC, source localization

\section{Introduction}

DOA estimation of sound sources is an important issue with many applications such as beamforming and speaker tracking. Real-world sound environments typically contain multiple directional sounds as well as diffuse noise, which comes from many directions like in vehicles or cafeterias. In this paper, we present crystal-MUSIC, a method for accurate estimation of the DOAs of multiple sources in the presence of diffuse noise.

One of the most fundamental approaches to DOA estimation is to maximize the output power of the delay-and-sum or other fixed beamformers with respect to the steering direction. Because of a broad beam pattern, however, DOA estimates by such methods may be inaccurate in the presence of multiple sources. Methods based on Time Delay Of Arrival (TDOA) estimates [1] widely in use today assume a single target source and again the performance can be degraded 
when more than one source is present. On the other hand, MUSIC [2-4] estimates the DOAs of multiple sources as directions in which the corresponding steering vector becomes most nearly orthogonal to the noise subspace.

It is important in MUSIC to accurately identify the noise subspace. When there is no noise, it is easily obtained as the null space of the observed covariance matrix. It can be obtained also in the presence of spatially white noise, since such noise only adds its power to all eigenvalues uniformly without changing the eigenvectors because of its spherical covariance structure. Therefore, the basis vectors of the noise subspace coincide with the eigenvectors of the observed covariance matrix belonging to the smallest eigenvalue. Directional noise can be dealt with as well, for it can be regarded as one of the directional signals. In contrast, diffuse noise can significantly degrade the identification of the noise subspace, because the noise spans the whole observation space, and unlike spatially white noise, its covariance structure is not spherical.

Aiming to make MUSIC robust to diffuse noise, this paper proposes a method for restoring the covariance matrix of directional signals from the observed covariance matrix contaminated by diffuse noise. The restoration is performed in two steps. In the first step, the contribution of diffuse noise is removed from the off-diagonal entries through the diagonalization of the covariance matrix of diffuse noise. This is performed through a technique of Blind Noise Decorrelation (BND) $[5,6]$, in which any covariance matrix of isotropic noise is diagonalized by a single unitary matrix based on the use of symmetrical arrays called crystal arrays. In the second step, thus obtained off-diagonal entries are completed to be the full matrix with the diagonal entries filled in via a low-rank matrix completion technique [7-9]. We present a modified version of the method in Ref. [7] with a positive semi-definite constraint on the estimated covariance matrix.

The rest of the paper is organized as follows. Section 2 gives a brief review of MUSIC, on which the proposed method is based. Section 3 describes our method for restoring the covariance matrix of the directional signals. Some experimental results are presented in Section 4, and the conclusion is stated in Section 5.

\section{Review of MUSIC}

We use the following notation throughout. The superscripts ${ }^{*}$ and ${ }^{\mathrm{H}}$ denote complex conjugation and Hermitian transposition, respectively. Signals are represented in the time-frequency domain with $\tau$ and $\omega$ denoting the frame index and the angular frequency. The covariance matrix of a zero-mean random vector $\gamma(\tau, \omega)$ is denoted by $\boldsymbol{\Phi}_{\gamma \gamma}(\tau, \omega) \triangleq \mathcal{E}\left[\gamma(\tau, \omega) \boldsymbol{\gamma}^{\mathrm{H}}(\tau, \omega)\right]$, where $\mathcal{E}[\cdot]$ is expectation.

\subsection{Observation model}

We assume that an array of $M$ microphones receives $L(<M)$ directional signals (some of them can be unwanted directional interferences) from unknown directions in the presence of diffuse noise. We assume the number of directional sources, $L$, to be known in this paper. Let $s(\tau, \omega) \in \mathbb{C}^{L}$ be the vector comprising the directional signals observed at a reference point (e.g. the array centroid), and $\boldsymbol{x}(\tau, \omega) \in \mathbb{C}^{M}$ and $\boldsymbol{v}(\tau, \omega) \in \mathbb{C}^{M}$ be the vectors comprising the observed signals and the diffuse noise at the microphones, respectively. Assuming planewave 
propagation and static sources of the directional signals, we can model the transfer function from $s_{l}(\tau, \omega)$ to $x_{m}(\tau, \omega)$ as $D_{m l}(\omega) \triangleq \mathrm{e}^{-\mathrm{j} \omega \delta_{m l}}$, where $\delta_{m l}$ denotes the delay in arrival of the directional signal $s_{l}(\tau, \omega)$ from the reference point to the $m$-th microphone. Consequently, our observation model is given by

$$
\begin{aligned}
\boldsymbol{x}(\tau, \omega) & =\boldsymbol{D}(\omega) \boldsymbol{s}(\tau, \omega)+\boldsymbol{v}(\tau, \omega) \\
& =\sum_{l=1}^{L} \boldsymbol{d}\left(\omega ; \theta_{l}\right) s_{l}(\tau, \omega)+\boldsymbol{v}(\tau, \omega),
\end{aligned}
$$

where $\theta_{l}$ denotes the DOA of the $l$-th directional sound,

$$
\boldsymbol{d}(\omega ; \theta) \triangleq\left[\mathrm{e}^{-\mathrm{j} \omega \delta_{1}(\theta)} \mathrm{e}^{-\mathrm{j} \omega \delta_{2}(\theta)} \ldots \mathrm{e}^{-\mathrm{j} \omega \delta_{M}(\theta)}\right]^{\top}
$$

denotes the steering vector corresponding to DOA $\theta$, and $\delta_{m}(\theta)$ denotes the time delay of arrival for DOA $\theta$ from the reference point to the $m$-th microphone. We assume $\boldsymbol{s}(\tau, \omega)$ and $\boldsymbol{v}(\tau, \omega)$ to be uncorrelated zero-mean random vectors. As a result, $\boldsymbol{x}(\tau, \omega)$ is a zero-mean random vector with covariance matrix

$$
\boldsymbol{\Phi}_{\boldsymbol{x} \boldsymbol{x}}(\tau, \omega)=\boldsymbol{D}(\omega) \boldsymbol{\Phi}_{\boldsymbol{s s}}(\tau, \omega) \boldsymbol{D}^{\mathrm{H}}(\omega)+\boldsymbol{\Phi}_{\boldsymbol{v} \boldsymbol{v}}(\tau, \omega) .
$$

\subsection{DOA estimation}

The orthogonal projection of $\boldsymbol{d}(\omega ; \theta)$ onto the noise subspace, i.e. the orthogonal complement of $\operatorname{span}\left\{\boldsymbol{d}\left(\omega ; \theta_{l}\right)\right\}_{l=1}^{L}$, becomes zero when $\theta$ coincides with $\theta_{l}$. Therefore, the MUSIC spectrum

$$
f_{\operatorname{MUSIC}}(\omega ; \theta) \triangleq\left\|\boldsymbol{V}^{\mathrm{H}}(\omega) \boldsymbol{d}(\omega ; \theta)\right\|_{2}^{-2}
$$

attains peaks at $\theta_{l}$, where $\boldsymbol{V}$ is a matrix whose columns are orthonormal basis vectors of the noise subspace. Since the MUSIC spectrum (5) is defined for each $\omega$, it is needed to integrate the information from all frequency bins in order to obtain a single estimate of the DOAs. A common approach is to average Eq. (5) over frequencies $[3,4]$. For example, the geometric mean [3] gives

$$
\bar{f}_{\mathrm{MUSIC}}(\theta) \triangleq\left[\prod_{\omega} f_{\mathrm{MUSIC}}(\omega ; \theta)\right]^{\frac{1}{K}},
$$

with $K$ denoting the number of averaged frequency bins. The DOAs are estimated as peaks in $\bar{f}_{\text {MUSIC }}(\theta)$.

\section{Denoising of the Spatial Covariance Matrix}

To calculate (5), it is important to accurately estimate $\boldsymbol{V}(\omega)$, namely basis vectors of the noise subspace. However, diffuse noise can significantly degrade the estimation, because it spans the whole observation space, and unlike spatially white noise, its covariance structure is not spherical. Our idea therefore consists in restoring the covariance matrix of the directional signals, $\boldsymbol{D}(\omega) \boldsymbol{\Phi}_{\boldsymbol{s} \boldsymbol{s}}(\tau, \omega) \boldsymbol{D}^{\mathrm{H}}(\omega)$, 
from the observed covariance matrix $\boldsymbol{\Phi}_{\boldsymbol{x} \boldsymbol{x}}(\tau, \omega)$ contaminated by diffuse noise, so that we can obtain $\boldsymbol{V}(\omega)$ as eigenvectors of the restored matrix belonging to the eigenvalue 0 . The matrix restoration consists of two steps. Firstly, the contribution of diffuse noise to the off-diagonal entries is removed using BND [5, 6] as explained in Section 3.1. Secondly, the diagonal entries are restored via a low-rank matrix completion technique [7-9] as explained in Section 3.2.

\subsection{Diffuse noise removal from the off-diagonal entries}

Coming from many directions, diffuse noise can be regarded as more isotropic than directional. Therefore, we make the following assumptions:

1) Diffuse noise has the same power spectrogram at all microphones:

$$
\left[\boldsymbol{\Phi}_{\boldsymbol{v v}}\right]_{11}(\tau, \omega)=\left[\boldsymbol{\Phi}_{\boldsymbol{v v}}\right]_{22}(\tau, \omega)=\cdots=\left[\boldsymbol{\Phi}_{\boldsymbol{v} \boldsymbol{v}}\right]_{M M}(\tau, \omega) .
$$

2) The inter-channel cross-spectrogram of diffuse noise is identical for all microphone pairs with an equal distance:

$$
r_{m n}=r_{p q} \Rightarrow\left[\boldsymbol{\Phi}_{\boldsymbol{v} \boldsymbol{v}}\right]_{m n}(\tau, \omega)=\left[\boldsymbol{\Phi}_{\boldsymbol{v} \boldsymbol{v}}\right]_{p q}(\tau, \omega),
$$

where $r_{m n}$ is the distance between the $m$-th and $n$-th microphones. It was shown that there exist such array geometries that any $\boldsymbol{\Phi}_{\boldsymbol{v} v}(\tau, \omega)$ satisfying these assumptions is diagonalized by a single unitary matrix $[5,6]$. So far, we have found five classes of geometries enabling such diagonalization, namely, regular polygonal, (twisted) rectangular, (twisted) regular polygonal prism, rectangular solid, and regular polyhedral arrays. They are called crystal arrays from their shapes.

Making use of a crystal array, we can remove the contribution of the diffuse noise to the off-diagonal entries as follows:

$$
\boldsymbol{P}^{\mathrm{H}} \boldsymbol{\Phi}_{\boldsymbol{x} \boldsymbol{x}}(\tau, \omega) \boldsymbol{P}=\boldsymbol{P}^{\mathrm{H}} \boldsymbol{D}(\omega) \boldsymbol{\Phi}_{\boldsymbol{s} \boldsymbol{s}}(\tau, \omega) \boldsymbol{D}^{\mathrm{H}}(\omega) \boldsymbol{P}+\boldsymbol{P}^{\mathrm{H}} \boldsymbol{\Phi}_{\boldsymbol{v} \boldsymbol{v}}(\tau, \omega) \boldsymbol{P}
$$

where $\boldsymbol{P}$ is a unitary diagonalization matrix of $\boldsymbol{\Phi}_{\boldsymbol{v} \boldsymbol{v}}(\tau, \omega)$.

\subsection{Restoration of the diagonal entries}

Now that the off-diagonal entries of $\boldsymbol{P}^{\mathrm{H}} \boldsymbol{D}(\omega) \boldsymbol{\Phi}_{\boldsymbol{s} \boldsymbol{s}}(\tau, \omega) \boldsymbol{D}^{\mathrm{H}}(\omega) \boldsymbol{P}$ has been obtained, the problem has reduced to that of completing its missing diagonal elements. Once this is done, the desired matrix $\boldsymbol{D}(\omega) \boldsymbol{\Phi}_{\boldsymbol{s} \boldsymbol{s}}(\tau, \omega) \boldsymbol{D}^{\mathrm{H}}(\omega)$ will be computed by the transformation $\boldsymbol{P}(\cdot) \boldsymbol{P}^{\mathrm{H}}$. Since $\boldsymbol{P}^{\mathrm{H}} \boldsymbol{D}(\omega) \boldsymbol{\Phi}_{\boldsymbol{s} \boldsymbol{s}}(\tau, \omega) \boldsymbol{D}^{\mathrm{H}}(\omega) \boldsymbol{P}$ is of rank at most $L$, the technique of low-rank matrix completion [7-9] can be applied. We present here a variant of an EM-based method by Srebro et al. [7] with a positive semi-definite constraint on the matrix to be completed. This is because MUSIC identifies the noise subspace based on the property that the eigenvectors of $\boldsymbol{D}(\omega) \boldsymbol{\Phi}_{\boldsymbol{s s}}(\tau, \omega) \boldsymbol{D}^{\mathrm{H}}(\omega)$ belonging to the positive and zero eigenvalues form bases of the signal and noise subspaces, respectively. Therefore, if the estimated matrix has some negative eigenvalues, there is no way of assigning the corresponding eigenvectors to one of these subspaces in a reasonable way.

We consider that we obtain via BND an imcomplete observation $\boldsymbol{Y}$ of $\boldsymbol{\Theta} \triangleq$ $\boldsymbol{P}^{\mathrm{H}} \boldsymbol{D}(\omega) \boldsymbol{\Phi}_{\boldsymbol{s s}}(\tau, \omega) \boldsymbol{D}^{\mathrm{H}}(\omega) \boldsymbol{P}$, where the obtained off-diagonal elements $\left\{x_{m n}\right\}$ are 
regarded as observables, and the missing diagonal elements $\left\{z_{m m}\right\}$ as latent variables. (Therefore, the diagonal entries of the basis-transformed observed covariance matrix $\boldsymbol{P}^{\mathrm{H}} \boldsymbol{\Phi}_{\boldsymbol{x} \boldsymbol{x}}(\tau, \omega) \boldsymbol{P}$ is just abandoned here.) The observation $\boldsymbol{Y}$ is considered to contain some errors because BND is generally not perfect. Therefore, $\boldsymbol{Y}$ is modeled as follows:

$$
\underbrace{\left[\begin{array}{cccc}
z_{11} & y_{12} & \cdots & y_{1 M} \\
y_{21} & z_{22} & \cdots & y_{2 M} \\
\vdots & \vdots & \ddots & \vdots \\
y_{M 1} & y_{M 2} & \cdots & z_{M M}
\end{array}\right]}_{\boldsymbol{Y}}=\underbrace{\left[\begin{array}{cccc}
\theta_{11} & \theta_{12} & \cdots & \theta_{1 M} \\
\theta_{21} & \theta_{22} & \cdots & \theta_{2 M} \\
\vdots & \vdots & \ddots & \vdots \\
\theta_{M 1} & \theta_{M 2} & \cdots & \theta_{M M}
\end{array}\right]}_{\boldsymbol{\Theta}}+\underbrace{\left[\begin{array}{cccc}
\epsilon_{11} & \epsilon_{12} & \cdots & \epsilon_{1 M} \\
\epsilon_{21} & \epsilon_{22} & \cdots & \epsilon_{2 M} \\
\vdots & \vdots & \ddots & \vdots \\
\epsilon_{M 1} & \epsilon_{M 2} & \cdots & \epsilon_{M M}
\end{array}\right]}_{\boldsymbol{E}},
$$

where $\boldsymbol{E}$ is the error term with $\epsilon_{m n}$ assumed to be i.i.d. complex-valued Gaussian random variables. The criterion is the maximization of the log-likelihood of the observed data subject to the constraint that $\Theta$ is positive semi-definite and of rank at most $L$ :

$$
\hat{\boldsymbol{\Theta}}=\arg \max _{\boldsymbol{\Theta} \in \Omega} \ln P\left(\left\{y_{m n}\right\}_{m \neq n} \mid \boldsymbol{\Theta}\right),
$$

where $\Omega$ denotes the set of the $M \times M$ positive semi-definite matrices of rank at most $L$.

The E-step amounts to the calculating the new estimate $\hat{\boldsymbol{Y}}^{(i+1)}$ of $\boldsymbol{Y}$ by completing the diagonal entries of $\boldsymbol{Y}$ by those of the current estimate $\hat{\boldsymbol{\Theta}}^{(i)}$ of $\boldsymbol{\Theta}$ :

$$
\hat{\boldsymbol{Y}}^{(i+1)}=\left[\begin{array}{cccc}
\hat{\theta}_{11}^{(i)} & y_{12} & \cdots & y_{1 M} \\
y_{21} & \hat{\theta}_{22}^{(i)} & \cdots & y_{2 M} \\
\vdots & \vdots & \ddots & \vdots \\
y_{M 1} & y_{M 2} & \cdots & \hat{\theta}_{M M}^{(i)}
\end{array}\right]
$$

The M-step amounts to calculating the new estimate $\hat{\boldsymbol{\Theta}}^{(i+1)}$ of $\boldsymbol{\Theta}$ as the best approximation of $\hat{\boldsymbol{Y}}^{(i+1)}$ in the Frobenius sense subject to $\boldsymbol{\Theta} \in \Omega$ :

$$
\hat{\boldsymbol{\Theta}}^{(i+1)}=\arg \min _{\boldsymbol{\Theta} \in \Omega}\left\|\hat{\boldsymbol{Y}}^{(i+1)}-\boldsymbol{\Theta}\right\|_{\mathrm{F}}
$$

We can write the solution to (13) explicitly using the eigenvalue decomposition of $\hat{\boldsymbol{Y}}^{(i+1)}$ :

$$
\hat{\boldsymbol{Y}}^{(i+1)}=\boldsymbol{U}^{(i+1)} \boldsymbol{\Lambda}^{(i+1)} \boldsymbol{U}^{\mathrm{H}(i+1)},
$$

where $\boldsymbol{U}^{(i+1)}$ is a unitary matrix and the eigenvalues in the diagonal of $\boldsymbol{\Lambda}^{(i+1)}$ are ordered from largest to smallest (possibly negative). Then, the solution to (13) is given by

$$
\hat{\boldsymbol{\Theta}}^{(i+1)}=\boldsymbol{U}^{(i+1)} \boldsymbol{\Lambda}_{\mathrm{T}}^{(i+1)} \boldsymbol{U}^{\mathrm{H}(i+1)} .
$$

Here, $\boldsymbol{\Lambda}_{\mathrm{T}}^{(i+1)}$ is the truncated version of $\boldsymbol{\Lambda}^{(i+1)}$ whose diagonal entry (eigenvalue of $\hat{\boldsymbol{Y}}^{(i+1)}$ ) is kept if and only if it is positive and among the $L$ largest and replaced by zero otherwise. 
The initialization is simply performed as follows: $\hat{\boldsymbol{Y}}^{(0)}=\hat{\boldsymbol{\Theta}}^{(0)}=\boldsymbol{P}^{\mathrm{H}} \boldsymbol{\Phi}_{\boldsymbol{x} \boldsymbol{x}} \boldsymbol{P}$. Using the resulting estimate $\hat{\boldsymbol{\Theta}}$, we obtain the estimate of $\boldsymbol{D} \boldsymbol{\Phi}_{\boldsymbol{s} \boldsymbol{s}} \boldsymbol{D}^{\mathrm{H}}$ as $\boldsymbol{P} \hat{\boldsymbol{\Theta}} \boldsymbol{P}^{\mathrm{H}}$, whereby obtaining the noise subspace $\boldsymbol{V}$ to be used in the calculation of the MUSIC spectrum (5).

\section{Experimental Results}

In this section, we present experimental results to show the effectiveness of crystal-MUSIC for the real-world noise and to compare it with conventional MUSIC. We used real-world noise recorded in a station building in Tokyo [10] with a square array of diameter $5 \mathrm{~cm}$. Two target speech signals were added to this noise recording under the assumption of plane wave propagation. The speech data were taken from the ATR Japanese speech database [11]. The duration of thus generated observed signals was $7 \mathrm{~s}$, and the sampling frequency was $16 \mathrm{kHz}$. We used STFT for subband decomposition, where the frame length and the frame shift were 512 and 64, respectively, and the Hamming window was used. $\boldsymbol{\Phi}_{\boldsymbol{x} \boldsymbol{x}}$ for both methods was calculated by averaging $\boldsymbol{x}(\tau, \omega) \boldsymbol{x}^{\mathrm{H}}(\tau, \omega)$ temporally over all frames.

As explained in Section 3, the main contribution of this paper is the restoration of the covariance matrix of the directional sounds from the observed covariance matrix. To see how well this technique works, we plot in Fig. 1 a relative Frobenius error defined by $\left\|\cdot-\boldsymbol{D} \boldsymbol{\Phi}_{\boldsymbol{s} \boldsymbol{s}} \boldsymbol{D}^{\mathrm{H}}\right\|_{\mathrm{F}} /\left\|\boldsymbol{D} \boldsymbol{\Phi}_{\boldsymbol{s} \boldsymbol{s}} \boldsymbol{D}^{\mathrm{H}}\right\|_{\mathrm{F}}$ as a function of the frequency. The solid and dashed lines are the results for the covariance matrices before the denoising: $\boldsymbol{\Phi}_{\boldsymbol{x} \boldsymbol{x}}$ and after the denoising: $\boldsymbol{P} \hat{\boldsymbol{\Theta}} \boldsymbol{P}^{\mathrm{H}}$, respectively. The SNR at the first microphone was adjusted to $-5 \mathrm{~dB}$ and the number of iterations of the EM algorithm was 100. The true DOAs of the target signals were $200^{\circ}$ and $260^{\circ}$. We see that the error was effectively reduced through the denoising.

From this result, an improved MUSIC spectrum is expected. To confirm this, we plot in Fig. 2 an example of (a) the conventional MUSIC spectra and (b) the crystal-MUSIC spectra, for each frequency. The SNR at the first microphone was adjusted to $-5 \mathrm{~dB}$ and the number of iterations of the EM algorithm was 100. The lines show the true DOAs of the target signals, namely $200^{\circ}$ and $260^{\circ}$. We see that the crystal-MUSIC gave more accurate peak positions and much less spurious peaks.

Finally, we compare the accuracy of DOA estimation by MUSIC and crystalMUSIC in a statistical manner. Fig. 3 shows the Root Mean Square Error (RMSE) of the DOA estimation by MUSIC and crystal-MUSIC as a function of the SNR at the first microphone. The DOA estimates were obtained from the geometric mean of narrowband MUSIC spectra as in Eq. (6). The range of averaging was 80th to 150 th frequency bins (approximately $2.5 \mathrm{kHz}$ to $4.7 \mathrm{kHz}$ ). The range was determined on the basis of the observation from Fig. 2 that, at the frequencies out of the range, the peaks were not reliable likely because of a low SNR and/or spatial aliasing. The RMSE was calculated from an experiment with various source DOAs, where all the 15 DOA combination from the set $\left\{0^{\circ}, 60^{\circ}, 120^{\circ}, \cdots, 300^{\circ}\right\}$ were tested. The figure shows a substantial improvement in RMSE by crystal-MUSIC. 


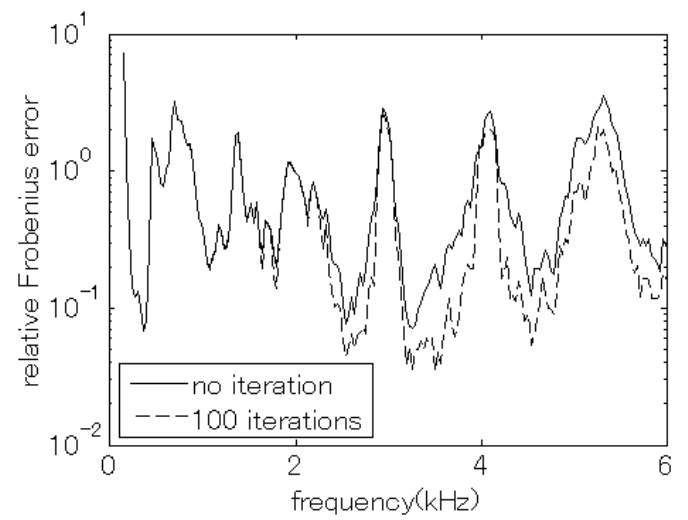

Fig. 1. The relative Frobenius error as a function of the frequency before and after the the covariance matrix restoration.

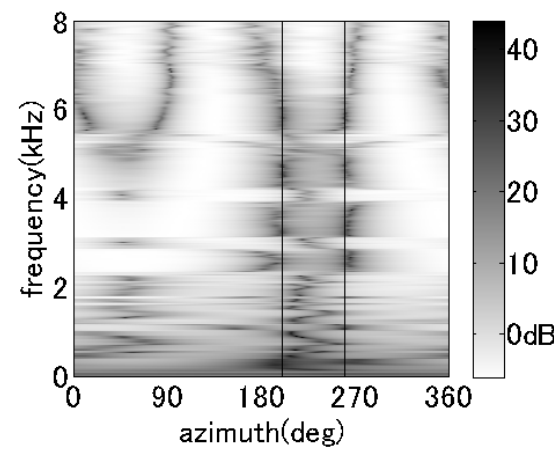

(a) subband MUSIC spectra

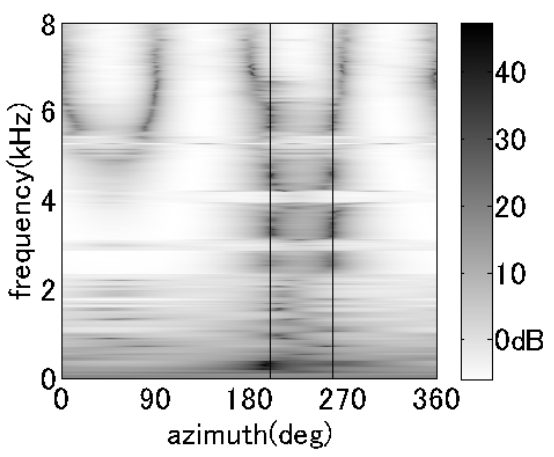

(b) subband crystal-MUSIC spectra

Fig. 2. An example of the subband MUSIC spectra for (a) conventional MUSIC and (b) crystal-MUSIC.

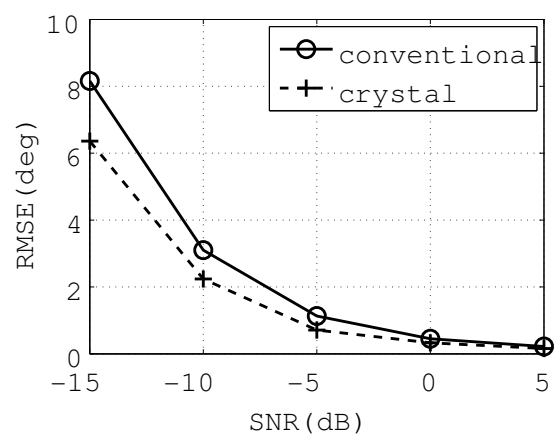

Fig. 3. The RMSE of DOA estimation as a function of SNR. 


\section{Conclusion}

We described crystal-MUSIC, an accurate method for estimating DOAs of multiple sounds in a diffuse noise field. It is based on removal of the contribution of diffuse noise from the observation covariance matrix via BND using crystal arrays and a low-rank matrix completion technique. We presented a new matrix completion method with a positive semi-definite constraint, which is more suitable to MUSIC. The experiment using real-world noise showed the effectiveness of the covariance matrix restoration and the substantial improvement in the DOA estimation accuracy by crystal-MUSIC.

Acknowledgments. This work is supported by INRIA under the Associate Team Program VERSAMUS and by Grant-in-Aid for Young Scientists (B)21760309 from MEXT, Japan.

\section{References}

1. C. H. Knapp and G. C. Carter, "The generalized correlation method for estimation of time delay," IEEE Trans. Acoust., Speech, Signal Process., no. 4, pp. 320-327, Aug. 1976.

2. R. O. Schmidt, "Multiple emitter location and signal parameter estimation," IEEE Trans. Antennas Propag., pp. 276-280, Mar. 1986.

3. M. Wax, T.-J. Shan, and T. Kailath, "Spatio-temporal spectral analysis by eignestructure methods," IEEE Trans. Acoust., Speech, Signal Process., pp. 817827, Aug. 1984.

4. T. Pham and B. M. Sadler, "Adaptive wideband aeroacoustic array processing," IEEE Trans. Acoust., Speech, Signal Process., pp. 817-827, Aug. 1984.

5. H. Shimizu, N. Ono, K. Matsumoto, and S. Sagayama, "Isotropic noise suppression in the power spectrum domain by symmetric microphone arrays," in Proc. WASPAA, New Paltz, NY, Oct. 2007, pp. 54-57.

6. N. Ono, N. Ito, and S. Sagayama, "Five classes of crystal arrays for blind decorrelation of diffuse noise," in Proc. SAM, Darmstadt, Germany, July 2008, pp. 151-154.

7. N. Srebro and T. Jaakkola, "Weighted low-rank approximations," in 20th International Conference on Machine Learning. AAAI Press, 2003, pp. 720-727.

8. E. J. Candès and B. Recht, "Exact matrix completion via convex optimization," The Journal of the Society for the Foundations of Computational Mathematics, no. 9, pp. 717-772, Apr. 2009.

9. S. Ji and J. Ye, "An accelerated gradient method for trace norm minimization," in Proceedings of the 26th International Conference on Machine Learning, Montreal, Canada, 2009, pp. 457-464.

10. N. Ito, N. Ono, E. Vincent, and S. Sagayama, "Designing the wiener post-filter for diffuse noise suppression using imaginary parts of inter-channel cross-spectra," in Proc. ICASSP 2010, Dallas, USA, Mar. 2010.

11. A. Kurematsu, K. Takeda, Y. Sagisaka, S. Katagiri, H. Kuwabara, and K. Shikano, "ATR Japanese speech database as a tool of speech recognition and synthesis," vol. 9, no. 4, pp. 357-363, Aug. 1990. 